ÉGYPTE monde arabe

\section{Égypte/Monde arabe}

21 | 1995

Économie égyptienne et perspectives de paix au Proche-Orient

\title{
Entre la communauté et la nation
}

Analyse de l'année politique selon l'Annuaire de l'Umma et le Rapport stratégique arabe

\section{Baudouin Dupret}

\section{(2) OpenEdition}

\section{Journals}

Édition électronique

URL : https://journals.openedition.org/ema/1711

DOI : $10.4000 /$ ema. 1711

ISSN : 2090-7273

Éditeur

CEDEJ - Centre d'études et de documentation économiques juridiques et sociales

Édition imprimée

Date de publication : 31 mars 1995

Pagination : 305-317

ISSN : 1110-5097

Référence électronique

Baudouin Dupret, « Entre la communauté et la nation », Égypte/Monde arabe [En ligne], 21 | 1995, mis en ligne le 08 juillet 2008, consulté le 07 juillet 2022. URL : http://journals.openedition.org/ema/1711 ; DOl : https://doi.org/10.4000/ema.1711

Ce document a été généré automatiquement le 7 juillet 2022

Tous droits réservés 


\title{
Entre la communauté et la nation
}

\author{
Analyse de l'année politique selon l'Annuaire de l'Umma et le Rapport \\ stratégique arabe
}

\section{Baudouin Dupret}

1 On a déjà eu l'occasion de faire état, par deux fois ${ }^{1}$, du pendant au fameux Rapport stratégique arabe édité chaque année par le Centre d'études stratégiques du journal AlAhrâm que constitue L'Annuaire de l'Umma (al-Umma fi 'âm)². La conclusion d'une de ces contributions soulignait toute l'utilité qu'il y aurait à comparer ces deux productions majeures de l'analyse politique égyptienne.

2 La tendance « Frères musulmans » de l'Annuaire fait peu de doutes. Il suffit, à cet égard, de parcourir la liste de ses collaborateurs. Par ailleurs, l'Annuaire de l'Umma fonctionne à la manière d'un important dossier de presse. L'information est, en effet, tirée pour l'essentiel des principaux quotidiens, hebdomadaires et mensuels égyptiens (Al-Ahrâm, Al-Akhbâr, Al-Gumhuryya, Al-Ahrâm al-iqtisâdî, Al-Musawwar, etc.) y compris les journaux de l'opposition (al-Sha'b, Al-Nûr, etc.).

3 Le Rapport stratégique, quant à lui, est bien davantage le produit d'un certain establishment académique. Fruit du fonctionnement d'une machine impressionnante (nombre de chercheurs et support financier), il se présente comme le produit d'une intelligentsia arabe pouvant à l'occasion se montrer critique. À mi-chemin entre la production scientifique et le journalisme analytique, il ne révèle pas aussi explicitement que l'Annuaire l'importance de son ancrage dans la presse égyptienne.

4 L'analyse comparative que l'on voudrait proposer tourne autour de deux réflexions. La première est inspirée des propos de 'Ali Dergham qui, dans sa courte présentation des deux premières éditions du Rapport ${ }^{3}$, faisait état du «besoin de formuler un nouveau modèle de pensée stratégique permettant de traiter de façon plus réaliste des problèmes du tiers-monde », besoin que le Rapport était censé combler. L'on pourrait légitimement se demander si cet objectif a été atteint, entre autres dans sa volonté de critiquer « le modèle occidental des études stratégiques » et dans « sa capacité à donner une explication scientifiquement valable des problèmes du tiers-monde $»^{4}$. Est-on vraiment en présence d'un modèle alternatif? 
Une autre question relève de l'inscription identitaire de chacune des deux publications. Comment, en effet, ne pas relever la mise en avant d'un référent islamique, celui de la Communauté (Umma) dans un cas, et celle du référent arabe dans l'autre? C'est sans doute ici que l'on trouvera la justification à la parution de deux ouvrages qui, annuellement, traitent sensiblement des mêmes questions et selon un canevas similaire, du moins si l'on s'en tient à la deuxième livraison de l'Annuaire. Chacun a la prétention d'offrir une lecture différente de la même actualité. Il reste toutefois à s'interroger sur cette spécificité revendiquée, sur sa portée... et ses limites. On doit ainsi se garder de sous-estimer l'inscription arabe de l'Annuaire. Il suffit, à cet égard, de noter que son sous-titre parle d'un rapport sur les questions arabes.

\section{Pour une nouvelle conception de la géopolitique?}

6 L'ambition de présenter un modèle alternatif arabe à la géopolitique dominante est-elle véritablement constitutive du Rapport et, dans sa propre version alternative, de l'Annuaire? Sans donner de réponse catégorique, quelques remarques peuvent toutefois être émises.

7 La première a trait au fait que les deux rapports stratégiques semblent sacrifier à la mode géopolitique contemporaine de l'analyse systémique. S'agissant de cette discipline (les deux publications étant mises de côté) et sans qu'il ne soit ici question d'en dénier les apports, l'on peut toutefois s'interroger sur la métaphore de la «forêt » symbolisant le système régional arabe, opposée aux «arbres » que constitueraient les États ${ }^{5}$, ainsi que sur les "particularités propres au sous-système arabe ${ }^{6}$. Le soussystème régional arabe existe-t-il vraiment? Son degré de "perméabilité » aux influences extérieures et la diversité qui le caractérise permettent-ils encore de parler d'une entité suffisamment homogène pour justifier qu'on la considère comme un tout?

Quelles que soient les réponses qu'il conviendrait d'apporter à ces interrogations, et pour en revenir au Rapport et à l'Annuaire, l'adhésion à ce modèle systémique des promoteurs de ces deux publications et des chercheurs qui y ont collaboré est patente. Il suffit, pour s'en rendre compte, de parcourir, à la table des matières de chacun des deux livres, les deux parties consacrées aux relations internationales. Dans toutes les éditions du Rapport, la première de ces deux parties s'intitule « Le système international et régional » et la seconde "Le système régional arabe ». Dans la première partie, il est question soit du "Moyen-Orient dans la politique internationale", des "relations régionales arabes ", du " conflit israélo-arabe » et de la "sécurité arabe » (1991); soit de « la sécurité arabe et de son environnement international et régional» (1992). Dans la seconde partie, il est question soit des " relations interarabes après la crise du Golfe " et de "peuple palestinien et lutte palestinienne» (1991), soit des «orientations principales des relations interarabes » et des « développements démocratiques dans le monde arabe » (1992).

9 L'Annuaire ne présente pas la même récurrence dans sa structure. Il faut ici rappeler qu'il est de parution bien plus récente, ce qui peut expliquer que son organisation ne soit pas encore parfaitement rodée. La première édition (1991) ne traite pas du système international. Cela ne la met pas à l'abri de l'explication systémique, puisqu'il y est question de «la diplomatie égyptienne dans le système régional». L'impact de ce dernier semble toutefois moins pris en considération, puisqu'il ne concerne qu'une seule des sept parties. II faut relever toutefois qu'il ne s'agissait, pour cette édition de 
1991, que d'un «rapport annuel sur les questions politiques et économiques égyptiennes ». Les choses changent avec la deuxième livraison de l'Annuaire (1992), qui semble davantage s'aligner sur le Rapport. Le livre aborde cette fois - glissement sémantique interne - les questions politiques et économiques arabes, et non plus seulement égyptiennes. La première partie est consacrée au "système international ", tandis que la seconde est consacrée au «monde arabe ». L'on doit ici noter un autre glissement sémantique, du Rapport à l'Umma cette fois, qui fait passer du "système » régional arabe au « monde » arabe.

10 Outre la prégnance de l'analyse systémique - élément que l'on ne saurait mettre au crédit d'une conception renouvelée de l'analyse stratégique -, il faut se demander dans quelle mesure le Rapport et l'Annuaire constituent véritablement des analyses stratégiques. On ne saurait dénier la pertinence de la prise en compte de facteurs internes aux différents États et sociétés dans une analyse stratégique régionale ou mondiale. Cela étant, il faut s'interroger sur la signification d'une concentration sur le local qui peut faire craindre une certaine dilution. Le Rapport, dans ses versions 1991 et 1992 qui sont fidèles au canevas d'origine, consacre sa troisième partie (entre 40 et $45 \%$ du nombre total de pages) à «la République arabe d'Égypte ». Un seul des quatre chapitres de cette partie concerne, à chaque fois, des questions à implications internationales, à savoir celui qui traite de « la politique extérieure égyptienne ». Tous les autres ont trait à la politique intérieure égyptienne, qu'il s'agisse du « système politique », de « la défense et les forces armées » ou de « l'économie égyptienne ». Cette remarque a déjà été formulée à maintes reprises, entre autres lors des colloques annuels consacrés à la discussion du Rapport stratégique (Nadwa munâqasha al-taqrîr alistrâtîjî̉ $)^{7}$. La part faite à l'Égypte est donc belle, sans que le stratégique - à moins de prendre le terme dans son sens le plus étendu - n'apparaisse toujours clairement.

11 La .critique est plus difficile à adresser à l'Annuaire 1991, qui ne parle que des « questions socio-économiques égyptiennes » : le rapport ne se prétend pas stratégique. Par contre, au vu de sa tendance croissante à se présenter en contrepoids du Rapport dans l'intégralité du spectre que celui-ci couvre et outre le fait qu'il affirme se consacrer aux questions socio-économiques arabes, l'Annuaire 1992 manifeste une évolution qui, pour inverse qu'elle soit de celle du Rapport (ce dernier a évolué vers un contenu plus « égyptien ", tandis que l'Annuaire évoluait vers un contenu plus « arabe » et « international »), ne le place pas moins dans le même champ d'intérêts et le soumet donc à une critique similaire. Ainsi, si l'édition 1991 consacrait ses sept parties (c'est-àdire la totalité du volume) à l'Égypte (le régime politique égyptien, les forces politiques de la société égyptienne, les forces du renouveau musulman, les groupes d'intérêts de la société égyptienne, les coptes dans la société égyptienne, la diplomatie égyptienne et le système régional et international, enfin l'économie égyptienne et les relations économiques internationales), il n'en va plus du tout de même dans l'édition 1992, qui ne consacre à l'Égypte que deux de ses quatre parties (le système international, le monde arabe, la République arabe d'Égypte et l'économie, soit $41 \%$ du nombre total de pages).

Que reste-t-il de l'originalité prétendue de l'analyse stratégique arabe ? Sans doute pas grand-chose en termes disciplinaires, mais certainement une production qui a trouvé une certaine cohérence et s'apparente, mutatis mutandis, à ce qu'une collection comme L'état $d u$ monde peut produire sous d'autres cieux. Au-delà d'un titre quelque peu trompeur et d'un certain effet de mode, le Rapport propose une lecture à la fois 
synthétique et digeste d'un ensemble de questions d'actualité récentes. Son pendant, l'Annuaire, a tendance à toujours davantage s'aligner, dans sa structure, sur ce canevas. L'originalité, dans un cas comme dans l'autre, relève donc davantage d'une formule annuelle de bilan international, régional et national établi par ce que l'Égypte produit d'intellectuels de tous bords, que du «nouveau modèle de pensée stratégique » dont parlait 'Ali Dergham.

\section{Lecture contrastée d'une même actualité} c'est à un autre niveau qu'il faudra chercher ce qui fart leur spécificité respective. C'est évidemment autour du référent idéologique (et donc identitaire) propre à chaque publication que s'articule la réponse. On fera ici quelques réflexions sur cette problématique avant d'illustrer le propos d'extraits de ces deux publications traitant de la violence politique et de la question sécuritaire.

etre le cheval de Troie de l'analyse des évolutions idéologiques de l'Égypte contemporaine - voire du monde arabe, mais on ne voudrait pas prêter le flanc à la critique d'une analyse trop unicitaire et systémique -, la problématique identitaire constitue un passage obligé, une sorte de question préjudicielle à laquelle une première réponse doit être apportée ${ }^{8}$. En la matière, un concept mis en évidence par Alain Roussillon semble particulièrement fécond: celui de convertibilité, qui tend à rendre compte d'un « système négatif fait d'interdits portant sur la remise en cause de valeurs ou d'options. [...] balisant le terrain de tout débat possible et définissant l'éventail des positions acceptables en matière d'élaboration théorique et idéologique $»^{9}$. Ce concept constitue sans doute l'une des clefs permettant de comprendre, par exemple, cet archétype de l'intellectuel que sa trajectoire a mené du nationalisme à l'islamisme ${ }^{10} \mathrm{et}$, partant, ce que cela peut signifier comme convergences sur nombre de questions. Ce passage du nationalisme à l'islamisme n'est d'ailleurs pas, si l'on en interroge les principaux tenants, le fruit d'une rupture dans l'univers sémantique et sémiotique qui précédait, mais bien le résultat d'une évolution, $d u$ franchissement d'une étape supplémentaire dans un processus dont la nature resterait fondamentalement identique. En interroger les principaux représentants, c'est «interroger le statut et les modalités des recompositions idéologiques au travers desquelles s'affirme la permanence de la visée identitaire [...] et qui commandent la production même du sens [...] et le système des relations des protagonistes en présence ${ }^{11}$.

Cette visée identitaire, on la retrouvera avec aisance en parcourant aussi bien le Rapport que l'Umma, le premier semblant plus directement constituer le prolongement d'une pensée nationaliste arabe telle que redéfinie par la nouvelle intelligentsia égyptienne, tandis que le second affirme explicitement son ancrage islamiste, mais à l'intérieur de l'espace de convertibilité que l'on a évoqué. C'est probablement ce même paramètre identitaire qui permet d'expliquer qu'au départ d'une même actualité, des lectures d'orientation différente puissent être données. Car, si le champ identitaire est clos, ce qu'il renferme n'en reste pas moins contrasté.

16 L'analyse proposée par chacune de ces deux publications (Rapport et Annuaire 1992) de la question sécuritaire en Égypte ${ }^{12}$ est, à cet égard, très significative. D'abord, dans la mesure du chapitre où il en est question. Alors que, très logiquement, l'Annuaire ne met pas en "situation de conjonction nécessaire " la violence politique et les mouvements

Égypte/Monde arabe, 21 | 1995 
islamistes, le Rapport ne manque pas d'opérer le rapprochement. C'est ainsi qu'en introduction au chapitre consacré à l'Alliance islamique et aux forces islamistes, l'auteur dit que «cette partie passera en revue le développement de l'Alliance islamique, qui rassemble le Parti socialiste du Travail, l'Association des Frères musulmans et le Parti libéral et s'étendra aussi, à la fin, sur les groupes islamiques pratiquant la violence et qui se sont montrés actifs au cours de l'année 1992 ».

L'on peut également relever des contestations importantes sur les sources d'information et leur utilisation. Le Rapport fait état d'une interview accordée par le porte-parole des Frères musulmans, Ma'mûn al-Hudaybî, à l'hebdomadaire français L'Express et rapportée par Rose al-Yûsuf, où il aurait dit : « Depuis trois mois, nous avons mis en garde les touristes et leur avons dit de ne pas approcher les régions de Louxor, Assouan et Dayrût. S'ils y vont, à eux d'en supporter les conséquences. Nous ne commettrons rien contre les étrangers, mais nous n'acceptons pas que qui que ce soit arbore des tenues légères, vienne chez nous boire du vin, consommer de la drogue et porter atteinte à l'honneur de l'islam ", alors que ses propos ont ensuite été réfutés. Mais le Rapport va plus loin puisqu'il affirme, peu après, que, « de toute manière, la position des Frères sur la question des touristes était empreinte d'une part de duplicité, sans doute pour conserver leur ligne traditionnelle qui cherche le maintien d'un équilibre entre, d'un côté, les groupes terroristes et, de l'autre, le régime ».

L'affaire Salsabîl, du nom d'une société informatique réputée proche de l'Association des Frères musulmans, qui a défrayé la chronique au début de l'année 1992 permet aussi d'identifier un certain nombre de clivages, le moindre n'étant sans doute pas que l'Annuaire, en dépit de sa présentation « objectivisante » des faits, est dans une certaine mesure partie au procès puisque explicitement apparenté aux Frères. Le Rapport est laconique et fait tout juste mention de "l'arrestation des membres d'une organisation secrète » qui utilisaient - le propos est attribué au ministre de l'Intérieur - le couvert d'une société informatique " pour exercer des activités politiques liées à l'association ». $\mathrm{Si}$ «les forces de sécurité emprisonnèrent nombre de personnes appartenant aux Frères tout comme elles investirent la société informatique Salsabîl, accusée d'exercer des activités politiques visant le régime en place », de leur côté, « les Frères récusèrent tout lien d'appartenance avec la société ». L'exposé de l'affaire et des prises de position est bien plus détaillé dans l'Annuaire. La part belle est faite à l'argumentaire des défenseurs des membres de la société poursuivis en justice. Il faut surtout pointer les remarques censées «introduire correctement cette affaire et en faire ressortir les tenants et aboutissants : $1^{\circ}$ ) les affaires traitées par la société Salsabîl ne concernaient pas exclusivement le secteur privé, bien au contraire [...]. Les activités et les opérations de la société avaient commencé, au grand jour, à fortement se développer [...]. $2^{\circ}$ ) II n'y avait aucune raison de craindre l'orientation islamique des propriétaires de cette société qui s'était spécialisée dans le secteur des données et du matériel informatiques. C'était une question sensible qui, nécessairement, suscitait la colère du pouvoir en place, lequel s'était engagé dans une confrontation d'une ampleur inhabituelle avec les groupements islamiques (Jamâ'ât islâmiyya) armés dans le but de mettre fin à l'usage de la violence contre les forces et les symboles du régime en place. $3^{\circ}$ ) L'information concernant la structure Salsabîl est allée de pair avec une information concernant le démantèlement d'une cellule d'espionnage israélienne dirigée par l'agent Fâris Misrâtî et sa fille Fâ'iqa Misrâtî. Cela a-t-il échauffé les esprits plus que de raison ou n'y avait-il là, pour l'appareil égyptien de la Sécurité, qu'une coïncidence?» 
Pour en terminer, l'on peut aussi relever l'explication contrastée donnée à la causalité de la violence politique qui secoue l'Égypte. Pour le Rapport, «la répartition géographique des activités des groupes violents se réclamant de l'islam politique révèle les dimensions socio-politiques sous-jacentes à l'explosion du phénomène et à sa recrudescence ces dernières années. Si les idées et les conduites adoptées par les groupes islamiques radicaux sont liées au type d'interprétation qu'ils font d'une pensée de référence, leur aptitude à développer et élargir leur champ d'action politique est très largement fonction des conditions sociales, économiques et culturelles prévalentes dans l'univers constituant leur environnement extérieur. [...] La plupart des études menées sur ces groupes montrent que l'immense majorité de leurs membres appartient à une tranche particulière de la société, à savoir la classe moyenne inférieure. Cette couche sociale se caractérise par sa grande tendance à l'instabilité politique. [...] De plus, cette couche sociale trouve ses origines parmi les émigrés ruraux qui sont arrivés récemment et souffrent des contradictions entre les valeurs traditionnelles de la campagne et les valeurs modernes citadines. [...] C'est cela surtout qui les a exposés aux difficultés propres à l'instabilité sociale qui accompagne le processus de "modernisation" rapide. Cette modernisation s'est toutefois opérée à un taux trop élevé par rapport à ce que pouvaient supporter ces villes. [...] L'ensemble de ces facteurs qui concourent à faire partir les gens et à les attirer en ville règlent, de manière informelle, le processus de l'émigration rurale. Cette dernière a exercé une pression constante sur les villes et particulièrement sur la capitale. [...] Toutes ces circonstances ont contribué à exacerber la frustration sociale de ces nouvelles classes d'origine rurale, dont les ambitions sociales et politiques s'étaient accrues au fil de leur vie citadine, et de la classe moyenne urbanisée pour qui l'émigration rurale constitua une pression perceptible dans les conditions de vie et de travail. Cela a ouvert la voie à de multiples manifestations d'instabilité sociale que les quartiers urbains informels incarnent de manière frappante (voir sur ce thème, dans le nº 20 d'Égypte/Monde arabe, l'analyse d'Eric Denis. NDLR). Ils sont devenus le terrain propice aux activités des groupes « se revendiquant de l'islam politique ».

L'Annuaire, pour sa part, met l'accent sur des éléments explicatifs bien différents, en tête desquels la relation quantitative et qualitative inverse qu'entretiennent sécurité politique et sécurité sociétale. «L'Égypte a été la proie, ces dernières années, de toutes sortes de méfaits sociaux. Au titre de ceux-ci, les sanglants affrontements entre les forces de sécurité et certains membres de groupes organisés s'opposant au régime. Il s'agit là d'un phénomène qui s'est répété dans plusieurs régions du pays, et particulièrement dans le Saïd. Avec pour résultat que les efforts de nature sécuritaire se sont portés sur l'élimination des périls menaçant la sécurité du régime. Ceci a conduit certains analystes à dire que la Sécurité égyptienne faisait passer en tête de ses préoccupations la sécurité politique du régime en place, loin devant la sécurité de la société. Pour bien saisir la nature de la relation qu'entretiennent sécurité politique et sécurité de la société, telle qu'elle ressort de différents cas concrets, l'on peut mettre l'accent sur différents points fondamentaux: 1) en dépit du fait que le budget du ministère de l'Intérieur ait dépassé, d'après les chiffres officiels avancés pour l'exercice 1990-91, l'ensemble des crédits financiers alloués à la santé et à l'enseignement réunis, les rapports de la Sécurité générale révèlent l'existence d'un affaiblissement du rapport entre le nombre de citoyens et la protection destinée à assurer leur sécurité [...], sans qu'il soit possible d'affirmer que la croissance démographique est la cause première de la régression des moyens mobilisés à cet effet. 2) Cette régression des moyens s'est 
accompagnée d'une augmentation du taux de criminalité en Égypte au cours des cinq dernières années. [...] 3) Les rapports publiés par les organisations de protection des droits de l'homme ont, d'un autre côté, fait état des sévices commis par les forces de sécurité contre les citoyens. [...] Il est inutile de dire qu'insister sur la distinction à opérer entre les événements liés à une sécurité qu'on pourrait qualifier de politicosociale et les événements qui dépassent ce cadre et modifient la nature des actions sécuritaires mène à rompre le cadre étroit de cette question sécuritaire, qu'il s'agisse de sédition confessionnelle ou d'autres incidents pour lesquels on utilise les vocables de "violence ", d'" extrémisme », de " terrorisme ». [...] Le fait que les aspects sécuritaires l'aient emporté est un des facteurs qui rendent chroniques ces événements. Il est impossible d'en sortir de la sorte. D'après nous, le dialogue, pour autant qu'on en suive les règles de base, permettrait peut-être d'identifier le nœud de la question ou, à tout le moins, de mettre en évidence les différentes tendances en présence.

\section{ANNEXES}

\section{Tables des matières du Rapport et de l'Annuaire, 1991 et 1992}

\section{Rapport 1991}

\section{Le système international et régional}

\subsection{Principaux développements de la politique internationale}

1.1.1. La désagrégation de l'Union soviétique et ses implications stratégiques

1.1.2. Émergence du rôle américain : phénomène et paradoxes

1.1.3. Développements stratégiques dans le monde arabe

1.1.4. Les Nations Unies et le règlement des conflits régionaux

1.2. Le Moyen-Orient dans la politique internationale

1.2.1. La sécurité dans le Golfe d'un point de vue américain

1.2.2. Le règlement du conflit israélo-arabe

\subsection{Les relations régionales arabes}

1.3.1. La chute du régime marxiste en Ethiopie et ses répercussions régionales

1.3.2. La sécurité du Golfe dans un cadre régional (points de vue iranien et turc)

1.3.3. Le problème kurde et les ambitions turques

1.3.4. La crise des otages et ses dimensions régionales

1.4. Le conflit israélo-arabe 
1.4.1. Les conflits de partis en Israël

1.4.2. Dimensions intérieures et extérieures de la question du règlement du conflit

1.4.3. Les développements de l'émigration juive

1.4.4. Les dimensions économiques de l'arrivée des immigrants

1.4.5. Les relations d'Israël avec les pays d'Europe de l'Est et l'ancienne Union soviétique

1.5. Les développements de l'économie internationale

1.6. La sécurité arabe

1.6.1. Les dimensions militaires du règlement du conflit israélien

1.6.2. Les développements de la défense des pays avoisinants

\section{Le système régional arabe}

2.1. Les relations interarabes après la crise du Golfe

2.1.1. Dislocation du système arabe et inexistence d'une alternative acceptable

2.1.2. Les questions politiques arabes après ta crise du Golfe

2.1.3. Développement interne des sociétés arabes (les organisations associatives et leur rôle dans le développement de la démocratie : Égypte et Tunisie)

2.2. Peuple palestinien et lutte palestinienne

2.2.1. Le peuple palestinien el le régime arabe

2.2.2. La position palestinienne dans le processus de paix

2.3. Les économies arabes

\section{La République arabe d'Égypte}

3. 1. Le système politique

3.1.1. Le régime politique

3.1.2. Les partis et les forces politiques

3.1.3. Les groupes d'intérêt

3.1.4. Les orientations de la presse égyptienne

3.1.5. Les incidents liés à la violence politique en Égypte

3.2. La défense et les forces armées

3.2.1. La politique de défense égyptienne

3.2.2. La politique d'armement égyptienne

3.2.3. La formation technique

3.2.4. La politique de service militaire pour la patrie

3.3. L'économie nationale

Égypte/Monde arabe, 21 | 1995 
3.3.1. Le programme de réforme économique

3.3.2. Le budget général de l'Etal

3.3.3. Le secteur public

\section{Rapport 1992}

\section{Le système international et régional}

1.1. Les principales forces de la politique mondiale

1.1.1. Les Nations Unies : les questions de paix et le règlement des cotisations

1.1.2. Les États-Unis d'Amérique

1.1.3. Le Commonwealth

1.1.4. L'Europe occidentale

1.1.5. Le conflit en Bosnie-Herzégovine

1.2. La sécurité arabe et son environnement international et régional

1.2.1. Le Proche-Orient et les engrenages stratégiques ayant mené à la fin de la guerre froide

1.2.2. Les relations et équilibres arabo-israéliens

1.2.3. L'Iran et les questions sécuritaires au Moyen-Orient

1.2.4. Le rôle de la Turquie au Moyen-Orient et les bénéfices qu'elle a tirés des développements régionaux et internationaux

1.2.5. La Corne de l'Afrique, entre chaos et réorganisation

\subsection{L'économie internationale}

\section{Le système régional arabe}

2.1. Les orientations principales des relations interarabes

2.1.1. Les nouveaux phénomènes dans les relations officielles arabes

2.1.2. La position arabe à l'égard des questions irakienne, libyenne et somalienne

2.1.3. La coordination arabe dans les négociations de paix

2.2. Les développements démocratiques dans le monde arabe

2.2.1. La situation du développement de la démocratie dans les pays arabes en 1992

2.2.2. Les élections qui se sont tenues dans le monde arabe en 1992

2.2.3. Les nouveaux mouvements islamiques dans le monde arabe

\section{La République arabe d'Égypte}

3.1. Le système politique

3.1.1. Le régime politique 
3.1.2. Les partis et les forces politiques

3.1.3. Les syndicats et les groupes de pression

3.1.4. Les orientations de la presse égyptienne

3.1.5. Tableau-catalogue des incidents liés à la violence politique et à la participation politique en Égypte

3.2. La défense et les forces armées

3.2.1. Les développements internationaux el régionaux et leurs répercussions sur la politique de défense égyptienne

3.2.2. La position stratégique des forces armées égyptiennes en 1992

3.2.3. La politique d'armement égyptienne

\subsection{L'économie égyptienne}

3.3.1. La mise en place de la politique de réforme économique

3.3.2. Les finances publiques

3.3.3. La politique monétaire el fiduciaire

3.3.4. La balance des paiements et la libéralisation du commerce extérieur

\section{Annuaire 1991}

\section{Le régime politique égyptien}

1.1. Le régime politique et la question des élections parlementaires

1.2. L'attitude des institutions du régime face à la crise du Golfe

\section{Les forces politiques de la société égyptienne}

2.1. Les forces de la vie politique égyptienne et te phénomène électoral

2.2. L'attitude des partis et des forces de l'opposition face à ta crise du Golfe

\section{Les forces du réformisme musulman}

3.1. Les Frères musulmans et les élections parlementaires

3.2. Les Frères musulmans el la crise du Golfe

3.3. Les caractéristiques générales du discours des Frères

\section{Les groupes d'intérêt de la société égyptienne}

4.1. Le syndicat des médecins

4.2. Le syndicat des ingénieurs

4.3. L'union générale des syndicats ouvriers d'Égypte

4.4. Les unions estudiantines au sein des universités égyptiennes 
4.5. Les clubs des membres du secteur de l'enseignement au sein des universités égyptiennes

4.6. L'Organisation égyptienne des droits de l'homme

\section{Les coptes dans la société égyptienne}

5.1. Les attitudes et préoccupations des coptes en Égypte

5.2. Les coptes et les élections

5.3. Le discours copte : tentative de renouveau

\section{La diplomatie égyptienne et le système régional et international}

6.1. La diplomatie égyptienne et la crise du Golfe

6.2. La diplomatie égyptienne et la question des armes de destruction massive dans la région du Proche-Orient

\section{L'économie égyptienne et les relations économiques internationales}

7.1. Aperçu des principaux développements de l'économie internationale

7.2. La production el les revenus nationaux en Égypte

7.3. Le budget général de l'État

7.4. La législation économique égyptienne

7.5. Statistiques économiques

\section{Annuaire 1992}

\section{Le système international}

Le nouvel ordre international et la région arabe

1.1. Les États-Unis dans le nouvel ordre international

1.2. Position de la région arabe dans la stratégie américaine après la guerre du Golfe

1.2.1. Les représentations stratégiques de la région après la guerre du Golfe

1.2.2. L'attitude américaine à l'égard des questions régionales les plus importantes

\section{Le monde arabe}

2.1. Le conflit arabo-israélien, le nouvel ordre international, la fin de l'histoire et l'homme

2.1.1. Le point de vue d'Israël face au nouvel ordre international

2.1.2. L'Intifada palestinienne

2.1.3. Nouvel ordre international et règlement pacifique du conflit arabo-israélien 2.2. La démocratie dans le monde arabe

2.2.1. Algérie

Égypte/Monde arabe, 21 | 1995 
2.2.2. Yémen

2.2.3. Soudan

2.2.4. Koweït

\section{La République arabe d'Égypte}

\subsection{Affaires extérieures}

3.1.1. Les négociations arabo-israéliennes

3.1.2. La crise entre la Libye et l'Occident

3.1.3. Les questions touchant à l'islam

3.2. Affaires intérieures

3.2.1. Les actes de l'Assemblée du Peuple

3.2.2. La politique de renseignement

3.2.3. Les désastres économiques et naturels

3.2.4. La politique sécuritaire et la question de la relation entre la sécurité politique et la sécurité sociétale

L'obligation absente, point de vue et récit d'une analyse culturelle de la sédition

\section{L'économie}

La reforme économique dans le cadre des politiques de libéralisation

\section{NOTES}

1. BURGAT F. et DUPRET B. (prés. et trad.), "L'Annuaire de l'Umma », Égypte-Monde arabe $n^{\circ} 11,1992$, pp. 183-192; DUPRET B. (prés. et trad.), «L'Annuaire de l'Umma et la question sécuritaire ", Égypte-Monde arabe $\mathrm{n}^{\circ}$ 17,1994, pp. 157-184.

2. Al'Umma fí 'âm. Taqrîr hawlî 'an al-shu'ûn al-siyâsiyya wa-l-iqtisâdiyya al-'arabiyya (La Nation en un an. Rapport politique et économique arabe), Dâr al-tabâ'a wa-l-nashr al-islâmiyya 1 (1990-91), Le Caire, Umma Press et Consultants arabes, 1992 et 2 (1991-92), Le Caire, Markaz al-dirâsât alhadâriyya, 1993.

3. AI-Taqrîr al-istrâtîjîl al-'arabî, 1 (1985) et 2 (1986), Markaz al-dirâsât al-siyâsiyya wa-l-istrâtîjiyya bi-l-Ahrâm, Le Caire.

4. DERGHAM A., «Les rapports stratégiques arabes de 1985 el 1986 », note de lecture, Bulletin du CEDEJ n 21,1987, pp. 217-219.

5. KORANY B., Les Régimes politiques arabes, Paris, PUF, 1990, p. 145.

6. Ibid, p.148.

7. Ainsi, par exemple, lors du colloque qui s'est tenu les 23 et 24/11/93 à l'Université du Caire, et qui traitait du rapport 1992.

8. Pour une introduction à la question, ainsi que pour la traduction d'un texte représentatif des dynamiques en présence, cf. B. DUPRET, «La problématique du nationalisme dans la pensée islamique contemporaine ", introd., et trad. de l'article de Rifat Sayyid Ahmad, Égypte-Monde arabe $n^{\circ} 15-16,1993$, pp. 377-425. 
9. ROUSSILLON A., «Égyptianité, arabité, islamité : la recomposition des référents identitaires », Égypte-Monde arabe $\mathrm{n}^{\circ}$ 11,1992, p. 129.

10. Cf. "Communisme, nationalisme, islamisme: itinéraire d'un intellectuel égyptien, 'Adil Husayn ", Égypte-Monde arabe ${ }^{\circ}$ 5,1991; "Tariq al-Bishri : les conditions d'un dialogue avec l'Occident ", Égypte-Monde arabe n 7, 1991; "Rashîd Ghannushi: islam, nationalisme, islamisme », Égypte-Monde arabe $\mathrm{n}^{\circ} 10,1992$.

11. ROUSSILLON A., op. cit., p. 83.

12. Pour la trad. complète des chapitres du Rapport et de l'Annuaire consacrés à la question, cf. $\mathrm{B}$. DUPRET (prés. et trad.), «L'Annuaire de l'Umma et la question sécuritaire » (cité) et « Le Rapport stratégique, l'islamisme et la violence politique », à paraître.

INDEX

Mots-clés : Frères musulmans, géopolitique

\section{AUTEUR}

\section{BAUDOUIN DUPRET}

CERMAC/Université de Louvain 